\title{
Metas de inflação e consistência expectacional em um modelo com regra de juros não linear ${ }^{*}$
}

\author{
Inflation targets and expectational consistency in a \\ model with a non-linear interest rule
}

\author{
Carlos Eduardo Iwai Drumond ${ }^{\dagger}$ \\ Cleiton Silva de Jesus ${ }^{\ddagger}$ \\ João Basilio Pereima ${ }^{\S}$
}

\begin{abstract}
Resumo
O principal objetivo deste artigo é construir um modelo macroeconômico de inspiração pós-keynesiana que leve em conta uma não-linearidade na regra de juros. Assume-se que a autoridade monetária considera, na determinação da taxa de juros, a interação entre a taxa de inflação e o nível de utilização da capacidade instalada, de modo que a sensibilidade da regra de juros ao hiato da inflação com relação à uma meta varia de acordo com o ciclo econômico. $\mathrm{O}$ arcabouço de política macroeconômica proposto no trabalho permite que a autoridade monetária possa dar peso tanto à inflação quanto ao produto sem perder de vista o papel de ancora expectacional da meta de inflação.
\end{abstract}

Palavras-chave: dinâmica pós-keynesiana; regra de juros não-linear; consistência expectacional.

\begin{abstract}
The aim of this paper is to develop a post-Keynesian macroeconomic model that takes into account a non-linearity in the interest rate rule. We assume that the monetary authority considers, in the practice of monetary policy, an interaction between inflation rates and the rate of capacity utilization, so that the sensitivity of the interest rate rule to the gap of inflation in relation to target varies according to the economic cycle. The macroeconomic policy framework proposed here allows the monetary authority to be sensitive to the inflation and to the output without, losing sight of the anchoring role of the inflation target.
\end{abstract}

Keywords: post-Keynesian dynamics; non-linear interest-rate-rule; expectational consistency.

JEL Classification: E00; E52; E58.

\footnotetext{
*Agradecemos aos precaristas anônimos pelas contribuições que ajudaram a melhorar a qualidade final do artigo. Esse artigo foi, em parte, financiado com recursos da Coordenação de Aperfeiçoamento de Pessoal de Nível Superior - Brasil (CAPES) - Grant Number 001.

† Professor do Departamento de Ciências Econômicas da UESC - ceidrumond@uesc.br

‡ Professor do curso de Ciências Econômicas da UEFS - cleiton.uefs@gmail.com

$\$$ Professor do curso de Ciências Econômicas da UFPR - joaobasilio@ufpr.br
} 


\section{Introdução}

Desde 1990 o regime de metas para a inflação (IT) passou a ser a norma de política monetária em vários bancos centrais ao redor do mundo. Atualmente cerca 35 países adotam este regime monetário (Schmidt-Hebbel e Carrasco, 2016), e vários são os estudos empíricos que avaliaram o desempenho macroeconômico dos países que têm seguido este arcabouço de política econômica (Ball e Sheridan, 2003; Gonçalves e Salles, 2008; Mendonça e Souza, 2012).

Mesmo a literatura macroeconômica convencional tendo avançado nas últimas duas décadas na construção de modelos teóricos que consideram explicitamente o regime IT (por exemplo, Clarida, Gali e Gertler, 1999), a literatura pós-keynesiana começou a considerar o arcabouço IT em modelos formais apenas recentemente, com os trabalhos seminais de Setterfield (2006) e Lima e Setterfield (2008). De fato, na sua forma original, o regime de metas de inflação deriva de um conjunto de hipóteses aparentemente antagônicas à tradição pós-keynesiana, especialmente no que diz respeito à ênfase dada à inflação baixa em detrimento de preocupações relacionadas com o nível do produto. Segundo Setterfield (2006), essa aparente incompatibilidade entre o regime IT e as preocupações keynesianas típicas poderia ser superada desde que: i) se considere devidamente o produto como parte primordial dos objetivos da política monetária; ii) o componente de conflito distributivo da inflação não seja negligenciado e; iii) se considere o papel da demanda agregada na determinação do produto real.

De fato, o debate sobre as causas da inflação não acontece sem controvérsias, especialmente quando se compara a literatura convencional com a abordagem pós-keynesiana. Como ressalta Montes (2009), dentro da tradição póskeynesiana, uma série de fatores são apontados como explicações possíveis para o movimento dos preços, de modo que se possa classificar a inflação de várias formas, tais como: i) inflação oriunda de pressões salariais, ii) inflação oriunda na variação das margens de lucro e do grau de monopólio, iii) variação dos preços oriunda de pass-through cambial, iv) inflação relacionada com diminuições nos retornos de escala, v) inflação oriunda exclusivamente dos movimentos na demanda, vi) inflação oriunda de mudanças na estrutura tributária e, finalmente, vii) inflação oriunda de choques de oferta.

A classificação feita anteriormente, obviamente, não encerra o debate sobre as causas da inflação e servem apenas como ponto de partida. Várias outras dimensões do processo inflacionário podem vir a tona, incluindo as expectativas dos agentes e o arcobouço institucional no qual esses agentes se inserem. Longe de 
encerrar o debate, contudo, é bastante usual, para fins de simplicidade analítica, classificar o processo inflacionário como advindos basicamente de duas grandes fontes, os custos e a demanda. Por outro lado, pode-se considerar, especialmente a partir das contribuições de Rowthorn (1977), que essas duas dimensões do processo inflacionário são, em última instância, relacionadas com o conflito distributivo, geralmente descrito como a disputa entre capitalistas e trabalhadores por frações da renda nacional. Embora o conflito distributivo seja mais evidente no caso da barganha por maiores salários, em vista de mudanças na conjuntura econômica, várias outras formas de conflito distributivo são bastante usuais, como, por exemplo, a tentativa de aumento das margens de lucro por parte das firmas ou mesmo o aumento dos impostos por parte do governo. Note-se que, embora essas mudanças nos preços sejam geradas por um componente de custos (mudança nos salários por exemplo), esses movimentos nos preços podem decorrer de reações endógenas à mudanças na demanda agregada/taxa de utilização da capacidade instalada. É basicamente esta abordagem a seguida neste trabalho, considera-se aqui o conflito distributivo, refletido na barganha salarial, como principal fonte de mudanças nos níveis de preços. Uma vez que os trabalhadores estão sujeitos à mudanças no cenário econômico que lhes permitem ter mais ou menos poder de barganha, a demanda agregada/taxa de utilização da capacidade instalada acaba por influênciar o movimento dos preços. A partir dos trabalhos de Setterfield (2006) e Lima e Setterfield (2008), alguns outros trabalhos passaram a incorporar, com diferentes estratégias de modelagem, a possibilidade de um regime de metas de inflação sob um framework fundamentalmente pós-keynesiano. Um traço comum desta literatura, tanto para economias fechadas quanto abertas, está na hipótese de linearidade da regra de política monetária, como pode ser verificado nos modelos dinâmicos desenvolvidos por Porcile, Souza e Viana (2011), Santos (2011), Drumond e Porcile (2012) e Drumond e Jesus (2016).

Quando se considera uma regra de política monetária linear, supõe-se implicitamente que seus coeficientes são constantes. Se este for o caso, a preocupação da autoridade monetária com a inflação, por exemplo, é sempre a mesma, independentemente se a economia estiver em expansão ou em contração. Refletindo acerca desta questão, Blinder (1997, p. 6) reconheceu que os macroeconomistas acadêmicos tendem a usar "funções de perda quadráticas por razões de conveniência matemática", (o que culmina em uma regra de política monetária linear) "sem pensar muito nas suas implicações substantivas". Além disso, estudos empíricos com dados de diferentes países têm sugerido que não são raros os casos em que os Bancos Centrais têm preferências assimétricas (Martin e Milas, 2004; Dolado et al., 2005; Surico, 2007; Cukierman e Muscatelli, 2008). 
Nessa perspectiva, não parece absurdo supor que os parâmetros de uma regra de política monetária variem ao longo do tempo de modo que dependam, por exemplo, de que fase do ciclo econômico a economia esteja.

Adicionalmente, uma outra questão substantiva surge quando o regime de metas de inflação é desenhado sob um framework pós-keynesiano. $\bigcirc$ foco concomitante na inflação e no produto só poderia ser plenamente consistente na presença de alguma política complementar à política monetária, como é o caso da política de rendas no modelo desenvolvido por Lima e Setterfield (2008). Ocorre que, como tentaremos demonstrar adiante, na ausência da hipótese de produto natural de longo prazo, assim como na ausência de políticas complementares à política monetária, uma regra de juros de duplo mandato típica não pode garantir a convergência da inflação efetiva para a meta de inflação.

Levando em conta as observações acima, o principal objetivo deste artigo é construir um modelo macroeconômico de inspiração pós-keynesiana que leve em conta uma não-linearidade na regra de juros. Consideraremos aqui que quanto mais próxima (afastada) do pleno nível de utilização da capacidade instalada a economia estiver, a autoridade monetária será mais (menos) sensível aos desvios da inflação com relação à meta. Esta dependência do parâmetro da inflação (na regra de juros) do nível de atividade econômica é uma clara distinção entre o modelo desenvolvido aqui e àqueles disponíveis na literatura pós-keynesiana até o presente momento.

Em termos de política macroeconômica, a principal contribuição do trabalho é apresentar uma regra de política monetária que, condicionada por limitações institucionais, especialmente no que diz respeito às políticas de renda, permita a autoridade monetária dar peso tanto à inflação quanto ao produto sem perder de vista o papel de ancora expectacional da meta de inflação. Não menos importante é o fato do modelo ser construído sem que se imponha de maneira ad hoc a convergência da inflação para a meta, ou se imponha alguma forma limite de comportamento expectacional, como é o caso da hipótese de expectativas racionais.

O presente artigo está organizado em mais três seções além desta introdução. Na segunda seção apresentamos uma breve revisão da literatura, na terceira seção propomos um modelo pós-keynesiano standard como ponto de partida da nossa análise e, finalmente, na quarta seção analisamos o modelo no contexto da regra de juros não linear. A conclusão encerra o trabalho. 


\section{Revisão da literatura e motivação}

Os principais ingredientes dos modelos pós-keynesianos que levam em conta o regime IT são os seguintes: i) o papel da demanda agregada é central na determinação da renda e do equilíbrio macroeconômico; ii) o mercado de bens opera abaixo do pleno emprego (existe capacidade instalada ociosa na economia); iii) a curva de Phillips é derivada a partir de um processo de barganha salarial; iv) a autoridade monetária manipula a taxa de juros para alcançar determinado(s) objetivo(s) macroeconômicos (a oferta de moeda é endógena); v) não existe taxa natural de juros; vi) as expectativas de inflação importam na determinação do nível observado de inflação. Além disso, considerações sobre políticas de rendas ${ }^{1}$, economia aberta e política fiscal também têm sido incorporadas em macro modelos pós-keynesianos sem muita dificuldade.

Sobre a curva Phillips, assim como em Setterfield (2006), diferentemente do que ocorre nas abordagens macro-monetárias convencionais, não é necessário assumir nenhum formato particular para ela, sobretudo, no que diz respeito ao longo prazo. Apesar disto, neste trabalho, considera-se que pressões inflacionárias advindas de mudanças no poder de barganha dos trabalhadores (que é endóngeno à mudanças na taxa de utilização da capacidade instalada) podem ocorrer fora do pleno emprego. A possibilidade de pressões inflacionárias antes que a economia se encontre no pleno emprego difere da visão difundida pela linha de pensamento conhecida como Modern Monetary Theory (MMT). Uma ampla discussão sobre a MMT pode ser encontrada em Wray (1998) e Wray (2014).

Nos modelos construídos por Setterfield (2006), Lima e Setterfield (2008) e Santos (2011), a compatibilização entre o regime IT e a macroeconomia póskeynesiana é possível porque existe um papel importante para as políticas de rendas, o que torna viável as autoridades políticas se preocuparem tanto com o nível de atividade quanto com a inflação e, ao mesmo tempo, garantirem a estabilidade do sistema econômico. De acordo com Setterfield (2006, p. 665) uma das peculiaridades de seu modelo pós-keynesiano estendido está no fato de que este modelo estrutural "involves policy making that explicitly recognizes the importance of aggregate demand conditions for real economic activity and the "conflicting claims" basis of the inflation process".

\footnotetext{
1 Setterfield (2007, p. 129) define políticas de rendas como sendo "formal and/or informal institutions that frame and mediate aggregate wage and price setting behaviour in such a way as to reduce conflict over income shares and better reconcile conflicting income claims". É neste sentido que a ideia de políticas de renda é entendida neste artigo.
} 
De fato, um dos principais resultados do modelo de Lima e Setterfield (2008) é que quanto mais ortodoxa for a combinação de políticas, mais adversas são as consequências para a estabilidade macroeconômica e a viabilidade do regime IT em um modelo pós-keynesiana. Já o modelo desenvolvido por Santos (2011), que é uma extensão do modelo de Lima e Setterfield (2008), sugere que, para que o equilíbrio dinâmico em uma economia pós-keynesiano seja estável, "the incomes policy should at least react to the inflation gap, while the monetary policy should at least react to the output gap" (Santos 2011, p. 316). Como pontua Carvalho (2015, p. 127) embora muitos seguidores de Keynes - incluindo alguns keynesianos da síntese neoclássica como Tobin (1985) - reconheçam que políticas de renda podem ser uma política de estabilização complementar, não é claro se Keynes proporia políticas de renda de maneira permanente. Na prática, a maioria das experiências com políticas de renda observadas no pós-guerra foi baseada no controle ou acompanhamento dos salários. No entanto, sabe-se que uma barreira para a implementação permanente das políticas de rendas nas economias capitalistas modernas é o arcabouço institucional da barganha salarial verificado em cada país. Diferenças no processo de moderação salarial e de barganha sindical podem inviabilizar do ponto de vista político a implementação de políticas de rendas para mediar o conflito distributivo e combater a inflação. Quanto maior for o país (e sua força de trabalho) e mais fragmentada for a sociedade (e os sindicatos), mais inviável se tornam as políticas de rendas.

Nesse sentido, uma alternativa ao uso das políticas de rendas como uma ferramenta de combate à inflação em um ambiente pós-keynesiano é considerar uma regra de juros linear ou IROP (Interest Rate Operating Procedure) que leve em conta tanto a inflação como o produto/emprego. É nesta perspectiva que são construídos os modelos de Porcile, Souza e Viana (2011), Drumond e Porcile (2012) e Drumond e Jesus (2016). Nestes trabalhos, implicitamente, assume-se que, embora o processo inflacionário seja oriundo dos custos (variações dos salários), a taxa de juros, ao influênciar a taxa de utilização da capacidade instalada, tenha condições de interferir na dinâmica dos preços. Sobre a capacidade dos juros impactarem a taxa de utilização da capacidade instalada e, consequentemente, a barganha salarial, segue-se nestre trabalho basicamente a mesma hipótese. Posto isto, os resultados do modelo devem ser interpretados circunscritos à esse cenário onde os juros influenciam o investimento e a taxa de utilização da capacidade instalada, como em Setterfield (2009) e Rochon e Setterfield (2006).

Nestes trabalhos, ao considerarem um conjunto de hipóteses fundamentalmente pós-keynesianas para pequenas economias abertas, é possível 
mostrar que a depender do arranjo de política macroeconômica considerado, o equilíbrio dinâmico derivado pelo modelo é estável, mesmo sem considerar qualquer tipo de políticas de renda. Por outro, como demonstraremos adiante, uma regra de juros linear deste tipo, apesar de gerar um equilíbrio dinâmico estável, gera uma importante inconsistência expectacional, ao não garantir a convergência da inflação efetiva para a meta.

Na seção seguinte procuramos descrever um modelo padrão que servirá de base para os exercícios subsequentes propostos no trabalho.

\section{Um modelo pós-keynesiano standard}

\subsection{O equilíbrio no mercado de bens e a curva IS}

Nesta seção procura-se construir um modelo standard de origem póskeynesiana considerando a existência de capacidade instalada ociosa na economia. Considere a seguinte demanda agregada para uma economia fechada

$$
Y=C+F+I
$$

Na qual $Y$ é o produto/demanda agregada, $C$ é o consumo agregado, $F$ é o gasto do governo e $I$ é o investimento agregado. $O$ consumo agregado é escrito como uma função linear da renda, de modo que $C=c Y$, com $0<c<1$. Por simplicidade e em vista dos objetivos deste trabalho, diferenças entre a propensão marginal a consumir de capitalistas e trabalhadores são desconsideradas.

Normalizando a demanda agregada em termos do estoque de capital obtémse

$$
u v=c u v+f+g
$$

Sendo $u=\frac{Y}{\bar{Y}}$ a taxa de utilização capacidade instalada, $\bar{Y}$ o produto de pleno emprego, v o inverso da relação capital produto, tomada como constante, c a propensão marginal a consumir, $\mathrm{f}$ o gasto do governo como proporção do estoque de capital e g o investimento como proporção do estoque de capital.

O investimento como proporção do estoque de capital, por seu turno, pode ser escrito como uma função positiva do nível de utilização da capacidade instalada e negativa da taxa real de juros

$$
\frac{I}{K}=g=g_{0}+\delta_{1} u-\delta_{2} r
$$


Sendo $g_{0}$ um parâmetro positivo que busca apreender o "espirito animal" dos empresários, $\mathrm{r}$ a taxa de juros real e $\delta_{1}$ e $\delta_{2}$ parâmetros positivos. A correta especificação de uma função investimento é motivo de controvérsia na literatura pós-keynesiana/kaleckiana, sendo que diferentes fechamentos no modelo podem ser obtidos a partir de distintas funções de investimento, como se pode ver em Setterfield (2017) e Hein, Lavoie e van Treeck (2011). Neste artigo, desconsideramos a eventual influência que uma taxa de utilização da capacidade instalada considerada normal pelos capitalistas poderia exercer na decisão de investir. Já o uso da taxa de juros na função investimento procura, de maneira análoga à feita por Setterfield (2009) e Rochon e Setterfield (2006), incorporar indiretamente o impacto dos juros sobre a taxa de lucro líquida auferida pelos capitalistas.

Tomando o equilíbrio no mercado de bens e substituindo a equação (2) na equação (3) é possível obter a seguinte curva IS normalizada em relação ao estoque de capital:

$$
u=\frac{g_{0}+f-\delta_{2} r}{v(1-c)-\delta_{1}}
$$

Valendo a condição keynesiana de equilíbrio $v(1-c)>\delta_{1}$, a curva IS é negativamente inclinada no plano taxa de utilização da capacidade instalada versus taxa de juros reais: uma variação positiva (negativa) da taxa de juros reais, tudo o mais constante, diminui (aumenta) o nível de utilização da capacidade instalada.

\subsection{Inflação, conflito distributivo e curva de Phillips}

Considerando um ambiente de concorrência imperfeita, no qual as firmas possuem poder de monopólio, a curva de Phillips dessa economia é construída a partir do conflito distributivo entre trabalhadores e capitalistas, em consonância com a tradição iniciada em Rowthorn (1977). Nesta economia em concorrência imperfeita a variação do nível de preços é igual à soma da variação dos salários nominais, da variação do mark-up das firmas e da produtividade do trabalho. Considerando, por simplicidade, o mark-up e a produtividade do trabalho como constantes, a inflação passa a ser explicada completamente pela variação nos salários nominais, que é descrito como uma função do hiato entre a participação dos salários na renda desejada pelos trabalhadores e a efetiva participação dos salários na renda definida pelo poder de mercado das firmas. Adicionalmente, os trabalhadores também levam em conta a inflação esperada no intuito de preservar os rendimentos reais. A partir dessas hipóteses, obtém-se a seguinte curva de Phillips: 


$$
\pi=\pi^{e}+\varnothing\left(w^{d}-w^{f}\right)
$$

Em que $\pi$ é a inflação efetiva, $\pi^{e}$ a inflação esperada e $w^{d}$ e $w^{f}$, respectivamente, a participação dos salários na renda desejada pelos trabalhadores e a participação dos salários na renda determinada pelos capitalistas, sendo $\emptyset$ um parâmetro estritamente positivo. $\bigcirc$ poder de barganha dos trabalhadores é modelado como uma função da taxa de utilização da capacidade instalada, de modo que $w^{d}=\alpha u$, sendo $\alpha$ um parâmetro positivo. Isso sugere que na medida em que a economia se encontra mais próxima do pleno uso da sua capacidade produtiva os trabalhadores têm mais poder de barganha e pressionam por maiores salários. Reescrevendo a equação (5):

$$
\pi=\pi^{e}+\varnothing\left(\alpha u-w^{f}\right)
$$

Note que o equilíbrio no processo de barganha salarial - igualdade entre a participação na renda desejada pelos trabalhadores e a efetiva participação dos salários na renda - implica que existe consistência expectacional, com a inflação efetiva sendo igual à inflação esperada pelos trabalhadores. Essa hipótese, contudo, não impõe nenhum modelo expectacional pré-determinado é plenamente compatível com comportamentos heurísticos dos agentes na tentativa de antecipar a inflação.

\section{Duplo mandato para a política monetária e consistência expectacional}

Tendo em vista a existência de uma relação negativa entre taxa de desemprego a taxa de utilização da capacidade, propõe-se uma regra de juros como função da inflação e da taxa de utilização da capacidade instalada. Em um primeiro momento, procura-se construir uma regra de juros linear típica, que leve em conta tanto a inflação como a taxa de utilização da capacidade instalada. Como se verá adiante, este tipo de regra, se incorporadas em um modelo pós-keynesiano, gera inconsistências expectacionais que precisam ser contornadas.

\subsection{Consistência expectacional e regra de juros}

Considere uma regra de juros dinâmica na qual a variação da taxa real de juros no tempo, $\dot{r}=\frac{d r}{d t}$, seja função linear do hiato entre a inflação observada e meta de inflação, bem como da diferença entre a taxa de utilização da capacidade instalada e o seu nível desejado pelas autoridades políticas. 


$$
\dot{r}=\gamma\left(\pi-\pi^{T}\right)+\beta\left(u-u^{T}\right)
$$

Sendo $\gamma$ e $\beta$ parâmetros positivos, na medida em que a inflação efetiva for superior/inferior à meta de inflação $\pi^{T}$ a taxa de juros real estará subindo/caindo. Por outro lado, a variação dos juros reais também responde ao hiato da taxa de utilização da capacidade instalada em relação a uma meta $u^{T}$ pré-estabelecida pelas autoridades políticas. Essa regra de juros pode ser modificada considerando regimes de política monetária exclusivamente focados na inflação (se $\beta=0$ ) ou exclusivamente preocupados com o produto/desemprego (se $\gamma=0$ ).

Uma vez definida a regra de juros, é preciso considerar o processo de convergência expectacional. Neste trabalho toma-se como referência uma dinâmica expectacional idêntica à apresentada por Tobin e Buiter (1976) e Turnovsky (1995), na qual os erros de previsão são continuamente revisados pelos agentes em um processo de aprendizado. Esse processo de aprendizado pode operar mesmo na presença de choques exógenos, uma vez que é baseado no desvio entre a inflação efetiva e a inflação esperada. No modelo aqui desenvolvido, por questões de simplicidade analítica, a existência deste tipo de choques não é explorada.

$$
\pi^{e}=k\left(\pi-\pi^{e}\right)
$$

A equação (8) descreve a dinâmica expectacional da inflação como uma resposta ao desvio da inflação efetiva da inflação esperada pelos agentes econômicos, sendo k>0 um parâmetro que mede a memória inflacionária. Uma das vantagens em usar essa equação é que não é preciso impor de maneira ex ante algum processo de ancoragem expectacional como, por exemplo, impor que a inflação necessariamente converge para alguma meta pré-estabelecida.

As equações (7) e (8) formam um modelo dinâmico bidimensional linear que pode ser analisado quando se combina a curva de Phillips, a curva IS, a regra de juros e a dinâmica expectacional. Após alguma manipulação algébrica, as dinâmicas da taxa de juros e da inflação esperada podem ser escritas da seguinte forma

$$
\begin{gathered}
\dot{r}=\gamma\left[\pi^{e}+\varnothing\left(\alpha\left(\frac{g_{0}+f-\delta_{2} r}{v(1-c)-\delta_{1}}\right)-w^{f}\right)-\pi^{T}\right]+\beta\left[\left(\frac{g_{0}+f-\delta_{2} r}{v(1-c)-\delta_{1}}\right)-u^{T}\right] \\
\pi^{e}=k \varnothing\left(\alpha\left(\frac{g_{0}+f-\delta_{2} r}{v(1-c)-\delta_{1}}\right)-w^{f}\right)
\end{gathered}
$$

A partir de então, é possível avaliar as propriedades dinâmicas do modelo tomando a matriz jacobiana do sistema formado pelas equações (9) e (10). 


$$
J=\left[\begin{array}{cc}
\frac{\partial \dot{r}}{\partial r} & \frac{\partial \dot{r}}{\partial \pi^{e}} \\
\frac{\partial \pi^{e}}{\partial r} & \frac{\partial \dot{\pi}^{e}}{\partial \pi^{e}}
\end{array}\right]
$$

É fácil notar que os elementos da matriz (11) são os seguintes:

$$
\begin{aligned}
& \frac{\partial \dot{r}}{\partial r}=\frac{-\delta_{2}(\emptyset \gamma \alpha+\beta)}{v(1-c)-\delta_{1}}<0 \\
& \frac{\partial \dot{r}}{\partial \pi^{e}}=\gamma>0 \\
& \frac{\partial \pi^{e}}{\partial r}=\frac{-k \emptyset \alpha \delta_{2}}{v(1-c)-\delta_{1}}<0 \\
& \frac{\partial \dot{\pi}^{e}}{\partial \pi^{e}}=0
\end{aligned}
$$

Com o traço de J negativo e o determinante de J positivo, pode-se concluir que o sistema dinâmico formado pelas equações (9) e (10) converge para um estado estacionário que é um ponto de equilíbrio estável.

$$
\begin{aligned}
& \operatorname{Det}(J)=\frac{k \emptyset \alpha \delta_{2} \gamma}{v(1-c)-\delta_{1}}>0 \\
& \operatorname{Tr}(J)=\frac{-\delta_{2}(\varnothing \gamma \alpha+\beta)}{v(1-c)-\delta_{1}}<0
\end{aligned}
$$

No estado estacionário a inflação esperada converge para a inflação efetiva, já que quando $\dot{\pi}^{e}=0$ então $\pi=\pi^{e}$. Isto implica, necessariamente, que a participação do salário na renda desejada pelos trabalhadores converge para a efetiva participação do salário na renda. Por outro lado, o modelo gera uma inconsistência da política monetária, já que, nada garante que a inflação esperada e a inflação efetiva convirjam para a meta de inflação determinada pela autoridade monetária.

Tome-se a equação (9) no estado estacionário $\operatorname{com} \pi=\pi^{e}$

$$
0=\gamma\left[\pi+\emptyset\left(\alpha\left(\frac{g_{0}+f-\delta_{2} r}{v(1-c)-\delta_{1}}\right)-w^{f}\right)-\pi^{T}\right]+\beta\left[\left(\frac{g_{0}+f-\delta_{2} r}{v(1-c)-\delta_{1}}\right)-u^{T}\right]
$$

Da equação (10), $\left(\alpha\left(\frac{g_{0}+f-\delta_{2} r}{v(1-c)-\delta_{1}}\right)-w^{f}\right)=0$ no estado estacionário. Logo, a equação (18) pode ser reescrita da seguinte forma 


$$
\gamma\left[\pi-\pi^{T}\right]=\beta\left[u^{T}-\left(\frac{g_{0}+f-\delta_{2} r}{v(1-c)-\delta_{1}}\right)\right]
$$

A partir da equação (19), pode-se perceber que, para dados valores de $\gamma$ e $\beta$, apenas no caso em que o governo consiga alcançar a meta para utilização da capacidade instalada é que a inflação convergirá para a meta de inflação (e viceversa). Não há, contudo, nenhum mecanismo endógeno ao modelo que garanta a igualdade de longo prazo entre $u$ e $u^{T}$ ou entre $\pi$ e $\pi^{T}$, de modo que alcançar concomitantemente as duas metas é algo completamente fortuito. Para o caso de economias abertas, resultados similares são encontrados nos modelos de Drumond e Porcile (2012) e Drumond e Jesus (2016).

Essa inconsistência entre a meta de inflação e a inflação efetiva, representa, certamente, um problema para a operacionalização da política monetária ao longo do tempo. Dada essa inconsistência pode-se pensar em duas alternativas opostas em termos de política monetária, um regime monetário exclusivamente focado na inflação (configurando $\beta=0$ ) e no outro extremo, um regime monetário exclusivamente focado na taxa de utilização da capacidade instalada (configurando $\gamma=0)$.

De fato, no caso do regime monetário exclusivamente focado na inflação, o sistema é estável e a economia converge para um estado estacionário onde não só a inflação é igual à inflação esperada, como também é igual à meta de inflação. $\bigcirc$ determinante da matriz jacobiana continua sendo o mesmo apresentado na

equação (16) enquanto o traço torna-se igual à $\frac{-\delta_{2}(\emptyset \gamma \alpha)}{v(1-c)-\delta_{1}}<0$. No caso do regime monetário exclusivamente focado na taxa de utilização da capacidade instalada $(\gamma=0)$ o modelo gera uma indeterminação dinâmica que não pode ser tomada como estabilidade, já que o determinante da matriz J torna-se nulo. Jesus e Correia (2016) desenvolvem um modelo macroeconômico com política fiscal ativa e chegam nesta mesma conclusão quando supõem que o movimento da taxa de juros em uma economia fechada depende exclusivamente do hiato do produto.

\section{Regra de juros não-linear}

O principal objetivo deste trabalho é propor uma regra de política monetária alternativa que, de um lado, torne viável um regime monetário de duplo mandato e, por outro lado, contorne o problema da inconsistência da política monetária apresentado anteriormente. No lugar de propor uma meta explícita para a taxa de 
utilização da capacidade instalada, procura-se neste trabalho propor uma regra de juros em que a sensibilidade da autoridade monetária aos desvios da inflação seja endógena e responda às mudanças na taxa de utilização da capacidade. Por um lado, quanto mais próxima de um estiver a taxa de utilização da capacidade instalada, mais sensível estará a autoridade monetária aos desvios da inflação com relação à meta. Por outro lado, quanto mais próximo de zero a autoridade monetária estará menos sensível. Essa regra de juros não linear é descrita pelo conjunto de equações abaixo:

$$
\begin{gathered}
\dot{r}=\gamma\left(\pi-\pi^{T}\right) \\
\gamma=\beta u \\
\dot{r}=(\beta u)\left(\pi-\pi^{T}\right), \quad \beta>0
\end{gathered}
$$

Na regra de juros da equação (22) não há uma meta explícita para a taxa de utilização da capacidade instalada, no entanto, a reação da política monetária ao hiato da inflação é não-linear de modo que, implicitamente, o produto de pleno emprego $\bar{Y}$ é tomado como alvo. Dito de outra maneira, a reação da política monetária ao hiato da inflação com relação a meta só será máxima somente quando o hiato do produto $(\ln Y-\ln \bar{Y})$ for nulo.

Resolvendo o modelo com essa nova regra de juros, obtém-se o seguinte sistema dinâmico bidimensional:

$$
\begin{gathered}
\dot{r}=\left(\beta\left(\frac{g_{0}+f-\delta_{2} r}{v(1-c)-\delta_{1}}\right)\right)\left[\pi^{e}+\emptyset\left(\alpha\left(\frac{g_{0}+f-\delta_{2} r}{v(1-c)-\delta_{1}}\right)-w^{f}\right)-\pi^{T}\right] \\
\pi^{e}=k \emptyset\left(\alpha\left(\frac{g_{0}+f-\delta_{2} r}{v(1-c)-\delta_{1}}\right)-w^{f}\right)
\end{gathered}
$$

O par de equações (23) e (24) formam um sistema dinâmico não-linear cuja estabilidade deve ser avaliada tomando as derivadas parciais das equações nos seus respectivos pontos estacionários:

$$
J=\left[\begin{array}{ll}
\left(\frac{\partial \dot{r}}{\partial r}\right)_{\left(r^{*}, \pi^{e^{*}}\right)} & \left(\frac{\partial \dot{r}}{\partial \pi^{e}}\right)_{\left(r^{*}, \pi^{e^{*}}\right)} \\
\left(\frac{\partial \pi^{e}}{\partial r}\right)_{\left(r^{*}, \pi^{e^{*}}\right)} & \left(\frac{\partial \pi^{e}}{\partial \pi^{e}}\right)_{\left(r^{*}, \pi^{e^{*}}\right)}
\end{array}\right]
$$

Avaliando as derivadas parciais nos pontos estacionários: 


$$
\begin{aligned}
\left(\frac{\partial \dot{r}}{\partial r}\right)_{\left(r^{*}, \pi^{e^{*}}\right)}= & -\left[\frac{\beta \delta_{2}}{(1-c)-\delta_{1}}\right]\left(\pi^{e}+\varnothing\left(\alpha \frac{g_{0}+f-\delta_{2} r^{*}}{v(1-c)-\delta_{1}}-w^{f}\right)-\pi^{T}\right) \\
& +\beta\left(\frac{g_{0}+f-\delta_{2} r^{*}}{v(1-c)-\delta_{1}}\right)\left(\frac{-\emptyset \alpha \delta_{2}}{v(1-c)-\delta_{1}}\right)
\end{aligned}
$$

$\operatorname{Com}\left(\pi^{e}+\varnothing\left(\alpha \frac{g_{0}+f-\delta_{2} r^{*}}{v(1-c)-\delta_{1}}-w^{f}\right)-\pi^{T}\right)=0$, obtêm-se:

$$
\begin{aligned}
& \left(\frac{\partial \dot{r}}{\partial r}\right)_{\left(r^{*}, \pi^{\left.e^{*}\right)}\right.}=-\beta\left(\frac{g_{0}+f-\delta_{2} r^{*}}{v(1-c)-\delta_{1}}\right)\left(\frac{\emptyset \alpha \delta_{2}}{v(1-c)-\delta_{1}}\right) \\
& \left(\frac{\partial \dot{r}}{\partial \pi^{e}}\right)_{\left(r^{*}, \pi^{\left.e^{*}\right)}\right.}=\beta\left(\frac{g_{0}+f-\delta_{2} r^{*}}{v(1-c)-\delta_{1}}\right) \\
& \left(\frac{\partial \dot{\pi}^{e}}{\partial r}\right)_{\left(r^{*}, \pi^{e^{*}}\right)}=\frac{-k \emptyset \alpha \delta_{2}}{v(1-c)-\delta_{1}} \\
& \left(\frac{\partial \dot{\pi}^{e}}{\partial \pi^{e}}\right)_{\left(r^{*}, \pi^{e^{*}}\right)}=0
\end{aligned}
$$

Note-se que $\frac{g_{0}+f-\delta_{2} r^{*}}{v(1-c)-\delta_{1}}=u^{*}$, onde $u^{*}$ é a taxa de utilização da capacidade instalada da economia no estado estacionário. Como a taxa de utilização da capacidade instalada é sempre um número positivo, tem-se que a derivada em (27) negativa e a derivada em (28) é positiva.

O traço da matriz jacobiana desta versão do modelo é igual à equação (27) e tem sinal negativo, enquanto o determinante da referida matriz é positivo, como descrito abaixo:

$$
\operatorname{Det}(J)=\beta\left(\frac{g_{0}+f-\delta_{2} r^{*}}{v(1-c)-\delta_{1}}\right) \frac{k \emptyset \alpha \delta_{2}}{v(1-c)-\delta_{1}}>0
$$

O resultado do modelo implica que a economia converge para um estado estacionário estável no qual a meta de inflação é alcançada de modo a não existir inconsistência intertemporal da política monetária. Também há coerência expectacional, sendo que a inflação efetiva converge para a inflação esperada que, por sua vez, converge para a meta definida pela autoridade monetária.

A proposição de uma regra monetária não-linear sugere a possibilidade de se incorporar uma preocupação com a taxa de utilização da capacidade instalada no processo de execução da política monetária sem, contudo, a necessidade de se incorporar políticas de rendas no modelo. Este resultado não invalida os esforços de formulação alternativa de política monetária que combinam meta de inflação e 
políticas de renda, como tem sido feito nos trabalhos de Setterfield (2006), Lima e Setterfield (2008) e Santos (2011). No entanto, acreditamos que o modelo aqui desenvolvido acrescenta à literatura pós-keynesiana uma opção alternativa de política monetária em modelos macrodinâmicos para economias fechadas, especialmente porque a regra de política monetária considerada neste artigo não é de difícil implementação nas economias capitalistas modernas.

\section{Conclusão}

A partir dos trabalhos de Setterfield (2006) e Lima e Setterfield (2008), uma série de trabalhos passou a considerar a possibilidade de compatibilizar o regime de metas de inflação com as hipóteses pós-keynesianas sobre o funcionamento das modernas economias. Uma das soluções nesta tentativa de compatibilização é a utilização das políticas de renda em complemento à política monetária.

Argumentamos nesse trabalho que, na ausência da viabilidade institucional de se utilizar de políticas de renda, uma forma alternativa de política deveria ser analisada. Uma opção, em particular, é a utilização de uma política monetária com regra de juros de duplo mandato, que considere tanto o hiato do produto quanto o hiato da inflação com relação à uma meta. Analisamos as características do modelo no contexto dessa regra de juros de duplo mandato linear e mostramos que essa regra, embora gere convergência para um estado estacionário estável, implica em uma inconsistência expectacional no longo prazo. Como demonstramos, na ausência da hipótese de produto natural de longo prazo, assim como na ausência de políticas complementares à política monetária, uma regra de juros de duplo mandato típica não pode garantir a convergência da inflação efetiva para a meta.

Uma vez feitas essas considerações, propusemos uma regra de juros não linear na qual a sensibilidade da política monetária ao hiato da inflação é sensível à taxa de utilização da capacidade instalada. Nesta nova formulação, quanto mais próxima (afastada) do pleno nível de utilização da capacidade instalada a economia estiver a autoridade monetária será mais (menos) sensível aos desvios da inflação com relação à meta. Mostramos que uma regra de política monetária deste tipo além de não ser de difícil implementação no mundo real, ela atende as condições de estabilidade de um modelo macrodinâmicos pós-keynesiano, de modo que a inflação converge para a meta de inflação no longo prazo mesmo quando a autoridade monetária se preocupa com a suavização do ciclo econômico.

Em termos de política macroeconômica, a principal contribuição deste trabalho é apresentar uma regra de política monetária que, condicionada por 
limitações institucionais, especialmente no que diz respeito às políticas de renda, permita a autoridade monetária dar peso tanto à inflação quanto ao produto/desemprego sem perder de vista o papel de ancora expectacional da meta de inflação. Novas pesquisas podem ser realizadas considerando outras nãolinearidades na regra de política monetária, ou até mesmo na curva de Phillips. Evidentemente, uma extensão natural deste trabalho consiste em verificar se as propriedades dinâmicas do modelo continuam válidas no caso de uma economia aberta ou quando se considera o papel ativo da política fiscal com uma restrição orçamentária do setor público.

\section{Referências}

Ball, L., \& Sheridan, N. (2003). Does Inflation Targeting Matter? (NBER Working Papers No. 9577). Cambridge, MA. https://doi.org/10.3386/w9577

Blinder, A. S. (1997). Distinguished Lecture on Economics in Government: What Central Bankers Could Learn from Academics-and Vice Versa. Journal of Economic Perspectives, 11(2), 3-19. https://doi.org/10.1257/jep.11.2.3

Carvalho, F. J. C. de. (2015). Liquidity Preference and Monetary Economies. London: Routledge. https://doi.org/10.4324/9781315734385

Clarida, R., Galí, J., \& Gertler, M. (1999). The Science of Monetary Policy: A New Keynesian Perspective. Journal of Economic Literature, 37(4), 1661-1707. https://doi.org/10.1257/jel.37.4.1661

Cukierman, A., \& Muscatelli, A. (2008). Nonlinear Taylor Rules and Asymmetric Preferences in Central Banking: Evidence from the United Kingdom and the United States. The B.E. Journal of Macroeconomics, 8(1), 2008. https://doi.org/10.2202/1935-1690.1488

Dolado, J. J., María-Dolores, R., \& Naveira, M. (2005). Are monetary-policy reaction functions asymmetric? The role of nonlinearity in the Phillips curve. European Economic Review, 49(2), 485-503. https://doi.org/10.1016/S0014-2921(03)00032-1

Drumond, C. E., \& De Jesus, C. S. (2016). Monetary and fiscal policy interactions in a post Keynesian open-economy model. Journal of Post Keynesian Economics, 39(2),

172-186. https://doi.org/10.1080/01603477.2016.1147332 
Drumond, C. E., \& Porcile, G. (2012). Inflation targeting in a developing economy: policy rules, growth, and stability. Journal of Post Keynesian Economics, 35(1), 137-162. https://doi.org/10.2753/pke0160-3477350108

Gonçalves, C. E. S., \& Salles, J. M. (2008). Inflation targeting in emerging economies: What do the data say? Journal of Development Economics, 85(1-2), 312-318. https://doi.org/10.1016/j.jdeveco.2006.07.002

Hein, E., Lavoie, M., \& van Treeck, T. (2011). Some instability puzzles in Kaleckian models of growth and distribution: a critical survey. Cambridge Journal of Economics, 35(3), 587-612. https://doi.org/10.1093/cje/beq026

Jesus, C. S., \& Correia, F. M. (2016). Active fiscal policy and macroeconomic stability. Journal of Economic Studies, 43(5), $749-762$. https://doi.org/10.1108/JES-03-2015-0052

Lima, G. T., \& Setterfield, M. (2008). Inflation targeting and macroeconomic stability in a Post Keynesian economy. Journal of Post Keynesian Economics, 3O(3), 435-461. https://doi.org/10.2753/PKE0160-3477300307

Martin, C., \& Milas, C. (2004). Modelling monetary policy: Inflation targeting in practice. Economica, 71(282), 209-221. https://doi.org/10.1111/j.00130427.2004.00366.x

Mendonça, H. F., \& de Guimarães e Souza, G. J. (2012). Is inflation targeting a good remedy to control inflation? Journal of Development Economics, 98(2), 178-191. https://doi.org/10.1016/j.jdeveco.2011.06.011

Montes, G. C. (2009). Reputation, credibility and monetary policy effectiveness. Estudos Econômicos (São Paulo), 39(3), 673-698. https://doi.org/10.1590/S0101-41612009000300009

Porcile, G., de Souza, A. G., \& Viana, R. (2011). External debt sustainability and policy rules in a small globalized economy. Structural Change and $\begin{array}{lll}\text { Economic Dynamics, 22(3), 269-276. } & \end{array}$ https://doi.org/10.1016/j.strueco.2011.06.002

Rochon, L.-P., \& Setterfield, M. (2007). Interest rates, income distribution, and monetary policy dominance: Post Keynesians and the "fair rate" of interest. Journal of Post Keynesian Economics, 30(1), 13-42. https://doi.org/10.2753/pke0160-3477300101

Rowthorn, R. (1977). Conflict, inflation and money. Cambridge Journal of Economics, 1(3), 215-239. https://doi.org/10.1093/oxfordjournals.cje.a035360 
Santos, A. L. M. (2011). Inflation targeting in a Post Keynesian economy. Journal of Post Keynesian Economics, 34(2), 295-318. https://doi.org/10.2753/PKE0160-3477340206

Schmidt-hebbel, K., \& Carrasco, M. (2016). The Past and Future of Inflation Targeting. In C. Ghate \& K. Kletzer (Eds.), Manuscript (pp. 583-622). New Delhi: Springer.

Setterfield, M. (2006). Is inflation targeting compatible with Post Keynesian economics? Journal of Post Keynesian Economics, 28(4), 653-671. https://doi.org/10.2753/pke0160-3477280407

Setterfield, M. (2007). The rise, decline and rise of incomes policies in the US during the post-war era: an institutional-analytical explanation of inflation and the functional distribution of income. Journal of Institutional Economics, 3(2), 127-146. https://doi.org/10.1017/S1744137407000665

Setterfield, M. (2009). Fiscal and monetary policy interactions: lessons for revising the EU Stability and Growth Pact. Journal of Post Keynesian Economics, 31(4), 623-643. https://doi.org/10.2753/pke0160-3477310406

Setterfield, M. (2017). Long-run variation in capacity utilization in the presence of a fixed normal rate, (New School for Social Research Working Papers No. 1704). https://doi.org/10.2139/ssrn.2929112

Surico, P. (2007). The Fed's monetary policy rule and U.S. inflation: The case of asymmetric preferences. Journal of Economic Dynamics and Control, 31(1), 305-324. https://doi.org/10.1016/j.jedc.2005.11.001

Tobin, J. (1985). Theoretical Issues in Macroeconomics. In G. Feiwel (Ed.), Issues in Contemporary Macroeconomics and Distribution (pp. 103-133). London: Palgrave Macmillan UK. https://doi.org/10.1007/978-1-349-06879-1_2

Tobin, J., \& Buiter, W. (1976). Long-run effects of fiscal and monetary policy on aggregate demand. In J. Stein (Ed.), Monetarism (pp. 273-336). Amsterdam: North Holland.

Turnovsky, S. (1995). Methods of Macroeconomic Dynamics. Cambridge, MA: MIT Press.

Wray, L. (1998) Understanding Modern Money. Cheltenham: Edward Elgar.

Wray, L. R. (2014). From the State Theory of Money to Modern Money Theory: An Alternative to Economic Orthodoxy (Levy Economics Institute of Bard College Working Paper No. 792). https://doi.org/10.2139/ssrn.2407711 